\title{
AFM investigation of hyaline cartilage's surface destruction
}

\author{
Mikhail Ihnatouski' ${ }^{1}$ Dmitriy Karev², Boris Karev ${ }^{3}$, Jolanta Pauk ${ }^{4}$, Kristina \\ Daunoravičien $\dot{e}^{5}$ \\ ${ }^{1}$ Yanka Kupala State University of Grodno, Belarus \\ ${ }^{2}$ Grodno State Medical University, Belarus \\ ${ }^{3}$ Grodno City Emergency Hospital, Belarus \\ ${ }^{4}$ Bialystok University of Technology, Poland \\ ${ }^{5}$ Vilnius Gediminas Technical University, Lithuania \\ E-mails: ${ }^{1}$ mii_by@mail.ru (corresponding author), ${ }^{2}$ dmitriy.karev@gmail.com, ${ }^{3}$ bkarev@gmail.com, \\ ${ }^{4}$ j.pauk@pb.edu.pl, ${ }^{5}$ kristina.daunoraviciene@vgtu.lt \\ (Received 30 March 2016; accepted 22 April 2016)
}

\begin{abstract}
Introduction: Osteoarthritis is a chronic, progressive disease. The aim of this paper is presenting the AFM investigation of cartilage in relation to the assessment of degenerative changes in the surface of hyaline cartilage. It can be useful in choosing the most effective methods of therapy.

Methods: Samples were taken from the cartilage surface of the femoral head after its removal during total hip arthroplasty. Images of the surface of the sample were obtained using an optical microscope equipped with a digital video camera, in the reflected light and by atomic force microscopy.

Results: The longitudinal orientation of the collagen fibers and sub-fibers beams on the surface, up to a diameter of $50 \mathrm{~nm}$ are identified in non-destroyed area sites.

Conclusions: Images of the destroyed areas displaying separately passing collagen fibers, strongly exposed to the surface: the size measured and found substructure.
\end{abstract}

Keywords: osteoarthritis, atomic force microscopy, hyaline cartilage.

\section{Introduction}

Osteoarthritis is a chronic, progressive disease which is characterized by an imbalance between the anabolic and catabolic processes in joint tissue [1]. Symptoms of osteoarthritis include the following: deep, achy joint pain exacerbated by extensive use, reduced range of motion and crepitus, frequently present, stiffness during rest [2]. The disease occurs in every third patient aged between 45 and 64 years and in 60-70\% patients over 65 years. Osteoarthritis primarily affects weight-bearing joints, which considerably impairs the quality of life of patients during the initial stages of its development, and ultimately leads to a disablement. A wide range of drugs' classes are used to treat osteoarthritis, although there is a lack of detailed guidance because of the variable nature of the condition. Despite the large amount of experimental and clinical data, no single scheme in the treatment and prevention of osteoarthritis is provided. Atomic force microscopy (AFM) has been widely used in studying nanostructure and nanomechanical properties of cartilage $[3,4]$. The aim of this paper is presenting the AFM of cartilage in relation to the assessment of degenerative changes in the surface of hyaline cartilage. It can be useful in choosing the most effective methods of therapy.

\section{Methods}

Tissue samples were taken from the cartilage surface of the femoral head after its removal during total hip arthroplasty were taken for nanostructure studies (Fig. 1a, b). Producing cartilage sample selection was based on initial visual inspection and evaluation of the degree of deformation of its surface was made. A sample intended for measurement was cut into a rectangular plate

(C) 2016 The Authors. Published by VGTU Press. This is an open-access article distributed under the terms of the Creative Commons Attribution License (CC-BY 4.0), which permits unrestricted use, distribution, and reproduction in any medium, provided the original author and source are credited. 
having a size of $\sim 10 \times 5 \mathrm{~mm}^{2}$. We tried to achieve flatness of platinum parties, protecting its edges so that they do not rise above its average level. The plate was fixed on a metal substrate. Images of the surface of the sample were obtained using an optical microscope "Micro $200 \mathrm{~T}-01$ ", equipped with a digital video camera, in the reflected light. Study of examples surface morphology was performed using an AFM NT-206 (CALC "Microtestmachines") in the static mode scan by a silicon cantilever $38 \mathrm{CSC}$. The processing and visualization was carried out by experimental software Surface Explorer (CALC "Microtestmachines") and nanoImages (CRCRSP of A. V. Luikov HMTI of the NPreliminary studies of the cartilage, which allow to determine the extent of the fracture surface, the presence of large parts of the morphology and the presence of artefacts were performed using an optical microscope at a magnification $100 \times, 200 \times, 500 \times 1000 \times$. These optical images are shown in Fig. 1b-e. The picture has no significant damage to the cartilage area (Fig. 1e, f) obtained at magnifications $200 \times$ and $500 \times$, you can find a variety of morphological objects, including rounded depressions ("gaps") in diameter from 3 to 16 microns; folds ("fibers") in width from 3 to 30 micrometers, laying in different directions; large surface protrusions. The area of the destroyed cartilage (Fig. 1d, f) obtained by increasing 200× and 1000×, morphology more monotonous. In addition to large surface protrusions, it is possible to consider displayed essentially in one-direction fibers. The fiber width is about 5 microns. The dry substance of cartilage should contain from 50 to $70 \%$ collagen and orientation of fibers is determined by the direction of lines of force arising from the deformation of the cartilage in the operation.

\section{Results}

Preliminary studies of the cartilage, which allow to determine the extent of the fracture surface, the presence of large parts of the morphology and the presence of artefacts were performed using an optical microscope at a magnification $100 \times, 200 \times, 500 \times 1000 \times$. These optical images are shown in Fig. 1b-e. The picture has no significant damage to the cartilage area (Fig. 1e, f) obtained at magnifications $200 \times$ and $500 \times$, you can find a variety of morphological objects, including rounded depressions ("gaps") in diameter from 3 to 16 microns; folds ("fibers") in width from 3 to 30 micrometers, laying in different directions; large surface protrusions. The area of the destroyed cartilage (Fig. 1d, f) obtained by increasing $200 \times$ and $1000 \times$, morphology more monotonous. In addition to large surface protrusions, it is possible to consider displayed essentially in onedirection fibers. The fiber width is about 5 microns. The dry substance of cartilage should contain from 50 to $70 \%$ collagen and orientation of fibers is determined by the direction of lines of force arising from the deformation of the cartilage in the operation.

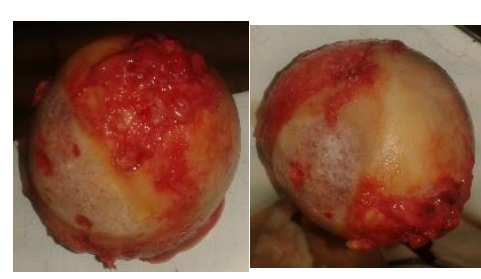

a)

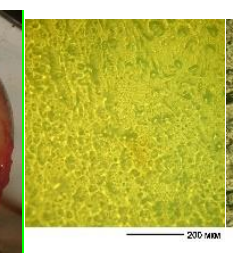

c)

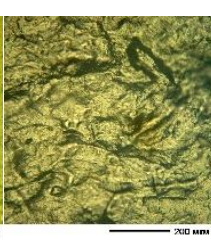

d)

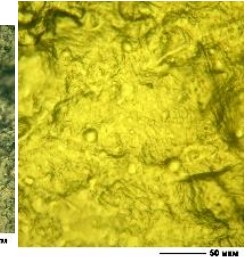

e)

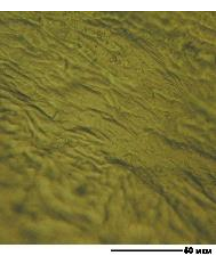

f)

Fig. 1. Optical image: a) and b) of the femoral head with strong destructions and with whole cartilaginous surfaces; c) and d) of the surface is not destroyed cartilage with increasing $200 \times$ and $500 \times$; d) and e) of the surface is destroyed $-200 \times$ and $1000 \times$

The AFM images of the surface of the cartilage not destroyed are presented in Fig. 2. Thus, it can be assumed that Fig. $2 \mathrm{f}$ displays a layer of one direction orientated fibers, exposed as a result of wear of the cartilage of the outer layers. Fig. $2 b$ shows the three-dimensional AFM image obtained with a relatively small magnification, the size of the surface area of the cartilage 
$15 \times 15 \mu \mathrm{m}^{2}$ with a maximum height difference of up to 1 micron. Irregularities of three cylindrical shapes are detected on the surface, which can be regarded as fiber. Cylinder axes are approximately parallel. The diameters of the fibers - about 4 microns. The complex structure of each fiber can be clearly observed. However, Fig. 2d shows a two-dimensional AFM images obtained at high magnification, the surface of the cartilage portion size $2 \times 2 \mu \mathrm{m}^{2}$ with a maximum height difference of up to $300 \mathrm{~nm}$.

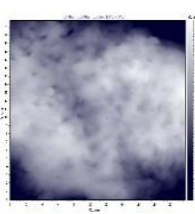

a)

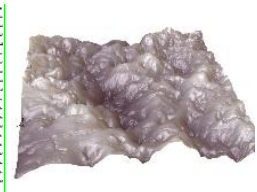

b)

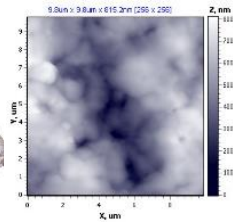

c)

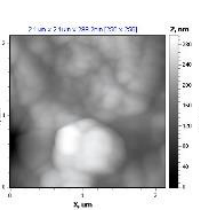

d)

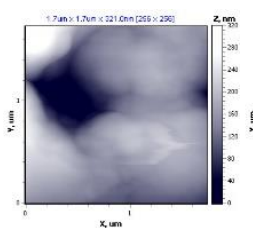

e)

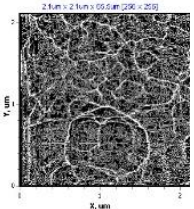

f)

Fig. 2. AFM images of the surface of the cartilage not destroyed: a) $21 \times 21$; b) $15 \times 15$; c) $10 \times 10$; d) $2,1 \times 2,1$; e) $1,5 \times 1,5$ and f) filtered images $2,1 \times 2,1 \mu \mathrm{m}^{2}$

It can be achieved a digital resolution up to $8 \mathrm{~nm} /$ pix with such aperture in the lateral plane. In the AFM image can be considered a complex fiber structure in detail. Laplace filter $3 \times 3$ has been applied for AFM images processing, contrasting gradient "gray" (Fig. 2f). Due to apply filters on a segmented AFM image clearly visible boundaries between the individual "sub-fibers" typical diameters range from 50 to $350 \mathrm{~nm}$. Interposition of sub-fibers and the presence of gaps between them are shown in Figure 2e with aperture $1.5 \times 1.5 \mu \mathrm{m}^{2}$. The AFM images of the surface of the cartilage destruction are presented in Fig. 3. A two-dimensional AFM image obtained by increasing the average, the size of the surface area of the cartilage in $7 \times 7 \mu^{2}$ with a maximum height difference of up to 2.5 microns is presented in Fig 3a. On the current image, scanning line intersecting the separate fiber lies about 3 microns wide. Likewise, the Fig. 3c shows threedimensional AFM image of the surface of the heavily damaged area of cartilage the size $18 \times 18$ $\mu \mathrm{m}^{2}$ with a maximum height difference of more than 2 microns. Almost half of the image (right end) has a level lower than the attitude of the measuring sensitivity of the device and presented at the AFM image in a monochromatic black field. The left edge in the image is occupied by two fibers having a developed surface structure (roughness). Fibers are directed along the diagonal of the image. Fig. 3b, e, f show profilograms' attitude measured along the scan line. On the left side in the Fig. 3d, e fiber's deformation can be seen, indicated by the arrow.

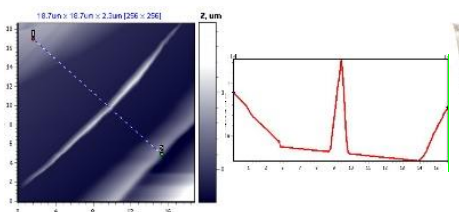

a)

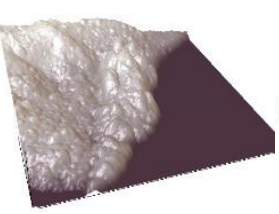

c)

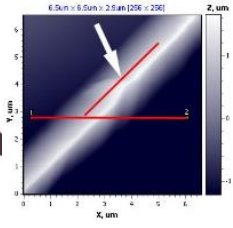

d)

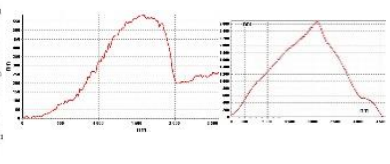

e) f)

Fig. 3. AFM images of the surface of the cartilage destruction: a) $18,2 \times 18,2 \mathrm{~mm}^{2}$; b) the profile of 13 microns, $1.8 \mathrm{~nm}$; c) $16 \times 16 \mu \mathrm{m}^{2}$; d) $6,4 \times 6,4 \mu \mathrm{m}^{2}$; e) profile of $2.2 \mu \mathrm{m}, 580 \mathrm{~nm}$, and f) the profile of $3.5 \mu \mathrm{m}$, $2500 \mathrm{~nm}$

\section{Conclusions}

Tissue samples during total hip arthroplasty were taken for elements of degradation of hyaline cartilage studies. Surface cartilage structures were detected by AFM. It is shown that the 
pathological destruction of cartilage leads to the impoverishment of a set of morphological elements its surface.

\section{References}

[1] Loeser, R. F. 2010. Age-related changes in the musculoskeletal system and the development of osteoarthritis, Clinics in Geriatric Medicine 26(3): 371-386.

http://dx.doi.org/10.1016/j.cger.2010.03.002

[2] Dieppe, P. 2010. Development in osteoarthritis, Rheumatology 50(2): 245-247. http://dx.doi.org/10.1093/rheumatology/keq373

[3] Wilusz, R. E. 2012. Immunofluorescence-guided atomic force microscopy to measure the micromechanical properties of the pericellular matrix of porcine articular cartilage, Journal of The Royal Society Interface 9(76): 2997-3007.

http://dx.doi.org/10.1098/rsif.2012.0314

[4] Zhu, P.; Fang, M. 2012. Nano-morphology of cartilage in hydrated and dehydrated conditions revealed by atomic force microscopy, Journal of Physical Chemistry \& Biophysics 2: 106.

http://dx.doi.org/10.4172/2161-0398.1000106 